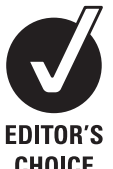

CHOICE

\title{
Unrecognised myocardial infarction and long-term risk of heart failure in the elderly: the Rotterdam Study
}

\author{
Maarten J G Leening, ${ }^{1}$ Suzette E Elias-Smale, ${ }^{1,2}$ Janine F Felix, ${ }^{1}$ Jan A Kors, ${ }^{3}$ \\ Jaap W Deckers, ${ }^{4}$ Albert Hofman, ${ }^{1}$ Bruno H Ch Stricker, ${ }^{1}$ Jacqueline C M Witteman ${ }^{1}$
}

\begin{abstract}
See Editorial, p 1434
${ }^{1}$ Department of Epidemiology, Erasmus MC, Rotterdam, The Netherlands

${ }^{2}$ Department of Radiology, Erasmus MC, Rotterdam, The Netherlands

${ }^{3}$ Department of Medical Informatics, Erasmus MC Rotterdam, The Netherlands ${ }^{4}$ Department of Cardiology, Erasmus MC, Rotterdam, The Netherlands
\end{abstract}

\section{Correspondence to}

Dr Jacqueline C M Witteman, Department of Epidemiology, Erasmus MC, P 0 Box 2040 3000 CA Rotterdam, The Netherlands; j.witteman@erasmusmc.nl

Accepted 6 April 2010 Published Online First 18 May 2010

\section{ABSTRACT}

Objective To examine the association between unrecognised myocardial infarction (MI) as detected by electrocardiography and the long-term risk of heart failure.

Design The Rotterdam Study is a prospective population-based cohort study of the general population of a suburb of the city of Rotterdam, The Netherlands.

Participants At baseline 2581 men and 3724 women aged $\geq 55$ years were classified on the basis of electrocardiography, interview and clinical data into those with recognised $\mathrm{Ml}$, those with ECG-based unrecognised $\mathrm{Ml}$ and those without Ml. The participants were followed-up for incident heart failure, death or end of the study period on 12 October 2006.

Results During a median follow-up time of 13.2 years, 823 cases of heart failure occurred, of which 403 in men. Independently of cardiovascular risk factors, recognised and unrecognised Mls yielded HRs of developing heart failure in men of 2.6 (95\% Cl 2.0 to 3.3$)$ and 2.1 (95\% Cl 1.5 to 2.9), respectively. In women, recognised $\mathrm{Ml}$ was associated with heart failure $(\mathrm{HR}=2.8 ; 95 \% \mathrm{Cl} 1.9$ to 4.1), whereas unrecognised MI was not significantly related to the risk of heart failure $(\mathrm{HR}=1.1 ; 95 \% \mathrm{Cl} 0.7$ to 1.7)

Conclusion Unrecognised Ml detected by electrocardiography yields a long-term risk of heart failure equivalent to recognised $\mathrm{Ml}$ in men, but is not significantly related to heart failure in women. In the light of the high incidence of both unrecognised $\mathrm{MI}$ and heart failure in the elderly, it may be worthwhile for both doctors and patients to improve responsiveness to typical and atypical symptoms of MI.

\section{INTRODUCTION}

A substantial proportion of all myocardial infarctions (MIs), particularly in the elderly, is asymptomatic or escapes clinical attention. This proportion ranges from $21 \%$ to $33 \%$ in men and $26 \%$ to $54 \%$ in women. ${ }^{1-7}$ Although many studies have shown that these so-called unrecognised MIs carry an equal or larger risk of cardiovascular and total mortality compared with recognised MIs, cardiovascular morbidity after unrecognised MIs has been little studied. ${ }^{3-6} 8$ Studies found that compared with recognised MI, unrecognised MI less often led to angina pectoris, and that it carried an equal risk of recurrent MI and a higher risk of cerebral small vessel disease and stroke in men. On the other hand, unrecognised MI was associated with a lower risk of recurrent MI and stroke in women. 5910 Only the Framingham Heart Study reported on the risk of heart failure, observing an increased risk of heart failure after unrecognised MI compared with recognised MI in men. No significant differences were found in women. ${ }^{6}$

Recent epidemiological studies have shown that the lifetime risk of developing heart failure for men and women of 50-55 years of age is $20-33 \%{ }^{11}{ }^{12}$ The incidence of heart failure shortly after a MI has increased over the past decades by $74 \%$, leading to steeply increasing rates of hospitalisation and associated costs. ${ }^{13} 14$ Moreover, heart failure is associated with a severe reduction in health-related quality of life, and life expectancy after the diagnosis of heart failure is substantially reduced, with a 5 -year mortality between $45 \%$ and $65 \% .^{121516}$

Coronary artery disease, and in particular MI, is known to be one of the most common causes of heart failure in the elderly. ${ }^{17-19}$ However, the longterm risk of heart failure after unrecognised MI has been studied less extensively, especially during the past decades, where extensive progression in cardiovascular treatment strategies has been made.

We examined the long-term risk of heart failure after unrecognised MI, detected by electrocardiogram (ECG), for men and women aged $\geq 55$ years in a prospective population-based cohort study with more than 13 years of median follow-up.

\section{METHODS}

\section{Study design, setting and population}

This study is part of the Rotterdam Study, a prospective, population-based cohort study of 7983 men and women aged $\geq 55$ years $(78 \%$ of the eligible population), living in a well-defined suburb of the city of Rotterdam, The Netherlands. The rationale and design of the study have been described elsewhere. ${ }^{20}$ Baseline data were collected from 1990 until 1993. Because our study focused on unrecognised MI at baseline and future risk of heart failure, we excluded people with prevalent heart failure at baseline $(n=248)$ and people who did not visit the research centre and therefore did not have an ECG recorded $(n=898)$. Thirty-six participants withdrew their informed consent for follow-up. Digitised ECGs or clinical data on MI status were missing in 496 participants, which left 6305 people eligible for this study. ECGs were missing owing to technical problems or lack of sufficient personnel to operate the ECG device.

The study was approved by the medical ethics committee of the Erasmus MC, Rotterdam, The Netherlands. Participants provided written informed consent to participate in the study and for information to be obtained from treating doctors 


\section{Assessment of MI by electrocardiography}

ECGs were processed by the Modular ECG Analysis System (MEANS) to obtain ECG measurement and interpretation. ${ }^{21}$ To determine MI, MEANS uses an extensive, but comprehensive, set of criteria that is derived from the Minnesota code. ${ }^{22}$ Subsequently, two research doctors blinded for other clinical information validated the ECGs selected by MEANS. A cardiologist, who specialised in ECG methodology, ascertained the final diagnosis of MI. The diagnosis of MI using MEANS is mainly driven by pathological $\mathrm{Q}$ waves and auxiliary criteria, such as $\mathrm{OR}$ ratio and $\mathrm{R}$-wave progression. ST-T changes were not considered as criteria for MI by MEANS, but were taken into account by the clinicians validating and ascertaining the diagnosis of MI based on ECG. Assessment of recognised MI status was done as reported previously for the Rotterdam Study. ${ }^{23}$ Based on this procedure, we classified participants at baseline as follows: a history of 'recognised MI' included people with selfreported MI confirmed by clinical data, with or without ECG characteristics matching a MI. A history of 'unrecognised MI' included all participants without documented or self-reported MI, but with ECG characteristics matching a MI. All unrecognised MIs were therefore Q-wave MIs. People without indication of MI on ECG and no medical documentation of an earlier MI, were classified as having 'no MI'.

\section{Assessment of covariates}

In the Rotterdam Study, assessment of anthropometrics, cardiovascular risk factors and use of drugs has been described previously. ${ }^{9}$ Diabetes mellitus was defined as a non-fasting serum glucose level $>11.0 \mathrm{mmol} / \mathrm{l}$ and/or the use of oral blood glucose-lowering drugs or insulin.

\section{Assessment of heart failure}

Prevalent heart failure at the baseline examination in the Rotterdam Study was assessed using a validated score based on the definition of heart failure of the European Society of Cardiology. ${ }^{24}$ Using this method, information on the presence of heart failure at baseline was available for all participants. Cases of incident heart failure were obtained by continuously monitoring participants of the Rotterdam Study during follow-up as described previously. ${ }^{12}$ The date of incident heart failure was defined as the day of the first occurrence of symptoms suggestive of heart failure, or the day of receipt of a first prescription for a loop diuretic or an ACE inhibitor, whichever came first. The diagnosis of heart failure was classified as definite, probable, possible, or unlikely. In accordance with the criteria of the European Society of Cardiology, only definite and probable cases were included in the analysis. A total of 169 possible and unlikely cases were not considered as heart failure events in the analysis. $^{12} 24$

\section{Statistical analysis}

All analyses were performed for men and women separately. Baseline characteristics were compared using Student $t$ test for continuous data and $\chi^{2}$ test for categorical data.

First, we constructed age-adjusted, heart failure-free survival curves using Cox proportional hazard analyses. Second, we used Cox proportional hazard analyses to calculate HRs for the risk of heart failure for recognised MI and unrecognised MI. ${ }^{25}$ We used the 'no MI' group as reference category. In model 1 we adjusted for age. In model 2, we additionally adjusted for the following cardiovascular risk factors: systolic blood pressure, diastolic blood pressure, use of antihypertensive drugs (defined as ACE inhibitors, $\beta$ blockers, diuretics, antiadrenergic agents, or calcium channel blockers), body mass index, diabetes mellitus, current and past smoking, serum total cholesterol and serum highdensity lipoprotein-cholesterol. Participants were censored at the time of occurrence of heart failure, death, loss to follow-up, or the end of the study period on 12 October 2006. In addition, we repeated all analyses, censoring participants when an incident MI occurred during follow-up. This was done to eliminate any possible distorting effects of incident MI during follow-up, since the risk of heart failure is highly elevated shortly after a MI. ${ }^{26}$ Approximately $5 \%$ of the participants had missing values for one or more cardiovascular covariates. These missing values were handled by single imputation using an expectation-maximisation algorithm. ${ }^{27}$ All measures of association are presented with $95 \%$ CIs. We used the level of significance of $p<0.05$ for all statistical analyses. Data were analysed using the SPSS statistical package, version 15.0 (SPSS Inc).

\section{RESULTS}

Table 1 shows the baseline characteristics of the study population for men and women separately. Women represented $59.1 \%$ of the participants. At the start of the study mean (SD) age was 67.8 years (8.0) for men and 69.3 years (9.2) for women. The 2581 men were classified as follows: 150 men had a previously unrecognised MI based on their ECG, 258 a recognised MI and 2173 no MI. For the 3724 women in the study the corresponding numbers were 177, 94 and 3453, respectively. People with a MI of any type were on average older, and had lower levels of serum high-density lipoprotein-cholesterol. Systolic and diastolic blood pressure was on average higher in the unrecognised MI group than in the other groups. In general, people with a recognised MI used antihypertensive drugs much more frequently. Smoking and diabetes mellitus was noticed more frequently in men with any type of MI in comparison with the no MI group. Women with unrecognised MI had on average a higher body mass index than in the other groups. During a median follow-up of 13.2 years (interquartile range $7.6-14.3$ years), eight participants were lost to follow-up, 2105 participants died, and 823 cases of incident heart failure occurred, of which 403 in men. The total number of heart failure events in each category of exposure is shown in table 2 .

Figure 1 displays age-adjusted, heart failure-free survival for men (figure 1A) and women (figure 1B). Cumulative incidences of heart failure after 15 years of follow-up were 17\% for no MI, $37 \%$ for unrecognised MI and 39\% for recognised MI in men. Women had lower cumulative incidences of heart failure of $11 \%$ for no MI, $13 \%$ for unrecognised MI, and $28 \%$ for recognised MI after 15 years of follow-up. Table 2 displays the results of the age-adjusted and multivariate-adjusted models for the risk of heart failure. Men with recognised MI or unrecognised MI had an age-adjusted HR for developing heart failure of $2.6(95 \% \mathrm{CI}$ 2.0 to 3.3 ) and 2.4 (95\% CI 1.7 to 3.3), respectively. Additional adjustment for cardiovascular covariates did not alter the estimate for recognised MI and slightly decreased the estimate for unrecognised $\mathrm{MI}(\mathrm{HR}=2.1 ; 95 \% \mathrm{CI} 1.5$ to 2.9). Women with a recognised $\mathrm{MI}$ had a nearly threefold increased age-adjusted risk of developing heart failure ( $\mathrm{HR}=2.8 ; 95 \% \mathrm{CI} 1.9$ to 4.1 ), which did not change after adjustment for cardiovascular risk factors. However, unrecognised MI in women was not significantly associated with age-adjusted risk for heart failure ( $\mathrm{HR}=1.2$; $95 \%$ CI 0.8 to 1.8$)$, or after additional adjustment for cardiovascular risk factors ( $\mathrm{HR}=1.1 ; 95 \%$ CI 0.7 to 1.7$)$.

When censoring 367 of the participants at the occurrence of an incident MI during follow-up, estimates of the HRs for recognised MI and unrecognised MI were virtually unchanged. 
Table 1 Baseline characteristics of the study population

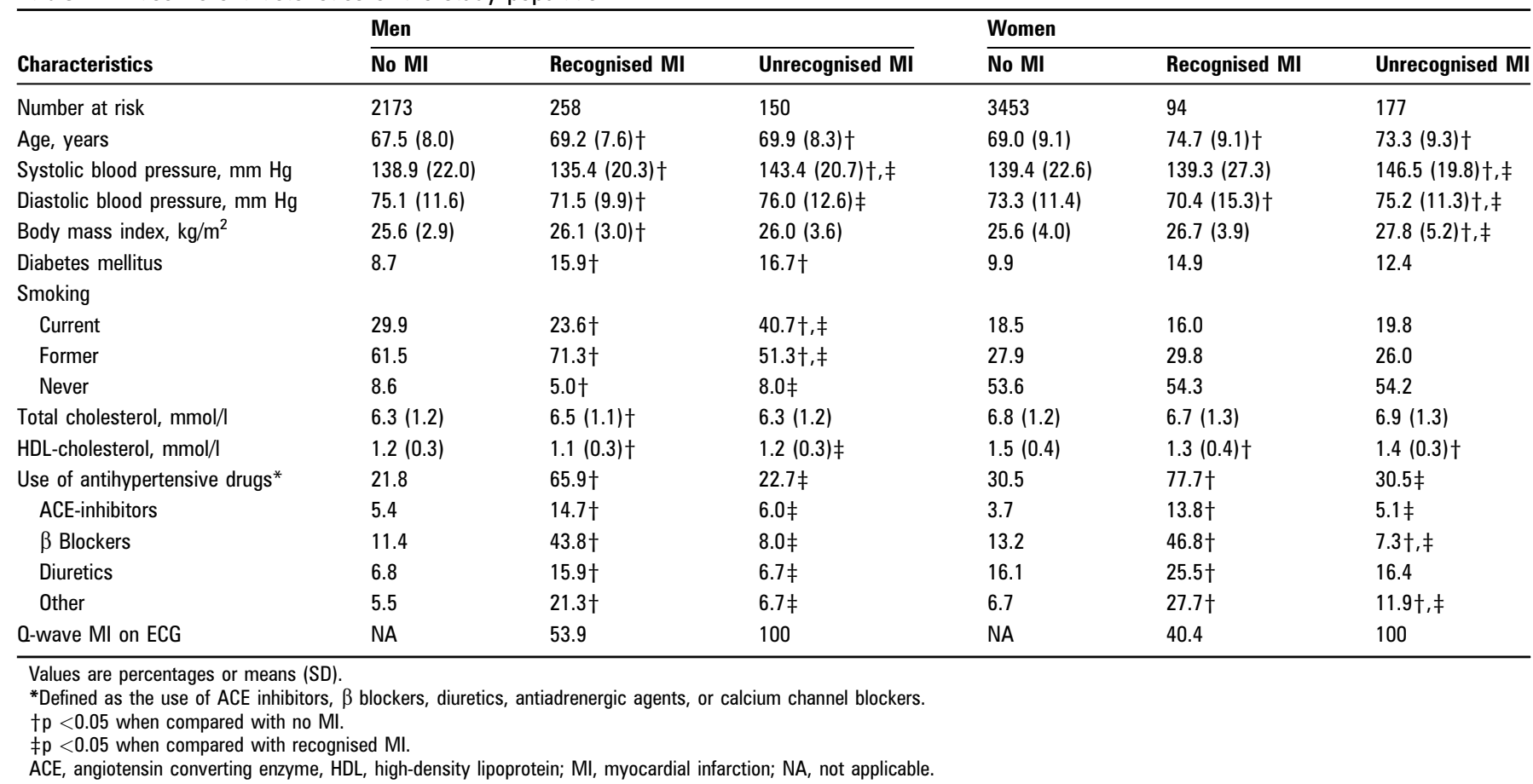

\section{DISCUSSION}

Our results indicate that in men the long-term risk of developing heart failure after an unrecognised MI, as detected by ECG, is equivalent to the long-term risk of heart failure after experiencing a recognised MI. Women with a recognised MI have a long-term risk of heart failure similar to men with a recognised MI, but women with unrecognised MI, as detected by ECG, do not seem to be more prone to the development of heart failure in later life.

\section{Previous studies}

In our study we focused on heart failure, whereas nearly all other studies on the prognosis of unrecognised MI examined a combination of cardiovascular outcomes. ${ }^{3-58}$ The Framingham Heart Study was the only one to evaluate heart failure after unrecognised MI as a separate outcome in the past. ${ }^{6}$ This study reported 10 year age-adjusted incidences of heart failure in men of $34.4 \%$ after unrecognised $\mathrm{MI}$ and $23.3 \%$ after recognised MI. In women, incidences of heart failure of $12.6 \%$ and $23.6 \%$ were found for unrecognised MI and recognised MI, respectively. These incidences seem to be in line with our findings, since in our study the 10-year age-adjusted incidences of heart failure in men were $23 \%$ and $25 \%$ for unrecognised MI detected by ECG and recognised MI, respectively. In women, incidences of $9 \%$ for unrecognised MI detected by ECG and 20\% for recognised MI were found. These similar incidences after MI are in accordance with a recent report from the Framingham Heart Study, indicating that the 5-year incidence of heart failure after surviving an initial MI beyond 30 days without heart failure has not changed over the past three decades. ${ }^{13}$

We found a HR of developing heart failure of 2.6 to 2.8 for recognised $\mathrm{MI}$ in men and women, respectively. The Cardiovascular Health Study reported on the relation between prevalent coronary heart disease and heart failure, indicating a nearly twofold increased risk. ${ }^{17}$ However, in this study coronary heart disease comprised recognised MI and also a history of angina pectoris and revascularisation procedures, which are less strongly associated with heart failure.

\section{Gender differences}

Our observation that ECG-based diagnosis of unrecognised MI is associated with an increased long-term risk of heart failure equivalent to that of recognised MI in men, but no increased risk in women is remarkable and needs some elaboration. This gender difference has been consistently described in other studies on the prognosis of unrecognised MI based on ECG investigating an array of cardiovascular outcomes-namely, stroke, cerebral small vessel disease, recurrent $\mathrm{MI}$ and death of cardiovascular origin. ${ }^{691028}$ A plausible explanation might be that especially in women ECG abnormalities not caused by coronary artery disease can be mistaken for MI because of difficulties with ECG lead placement owing to breast tissue. ${ }^{28}$ This misclassification

Table 2 Hazard ratios (HRs) for developing heart failure

\begin{tabular}{|c|c|c|c|c|c|c|c|c|}
\hline & \multicolumn{4}{|l|}{ Men } & \multicolumn{4}{|c|}{ Women } \\
\hline & $\mathbf{N}$ & $\mathbf{n}$ & $\begin{array}{l}\text { Model 1† } \\
\text { HR (95\% Cl) }\end{array}$ & $\begin{array}{l}\text { Model 2‡ } \\
\text { HR (95\% Cl) }\end{array}$ & $\mathbf{N}$ & $\mathbf{n}$ & $\begin{array}{l}\text { Model 1† } \\
\text { HR }(95 \% \mathrm{Cl})\end{array}$ & $\begin{array}{l}\text { Model 2‡ } \\
\text { HR (95\% CI) }\end{array}$ \\
\hline No Ml & 2173 & 282 & 1.0 (reference) & 1.0 (reference) & 3453 & 367 & 1.0 (reference) & 1.0 (reference) \\
\hline Unrecognised MI & 150 & 41 & $2.4(1.7 \text { to } 3.3)^{*}$ & $2.1(1.5 \text { to } 2.9)^{*}$ & 177 & 26 & $1.2(0.8$ to 1.8$)$ & 1.1 (0.7 to 1.7$)$ \\
\hline
\end{tabular}

${ }^{*} \mathrm{p}<0.001$.

†Adjusted for age.

$\ddagger$ Adjusted for age, systolic blood pressure, diastolic blood pressure, antihypertensive drugs, body mass index, diabetes mellitus, smoking, total cholesterol and HDL-cholesterol.

$\mathrm{HDL}$, high-density lipoprotein; MI, myocardial infarction; N, number at risk; n, number of events. 
Figure 1 (A) Age-adjusted heart failure-free survival curves for men. (B) Age-adjusted heart failure-free survival curves for women. MI, myocardial infarction.
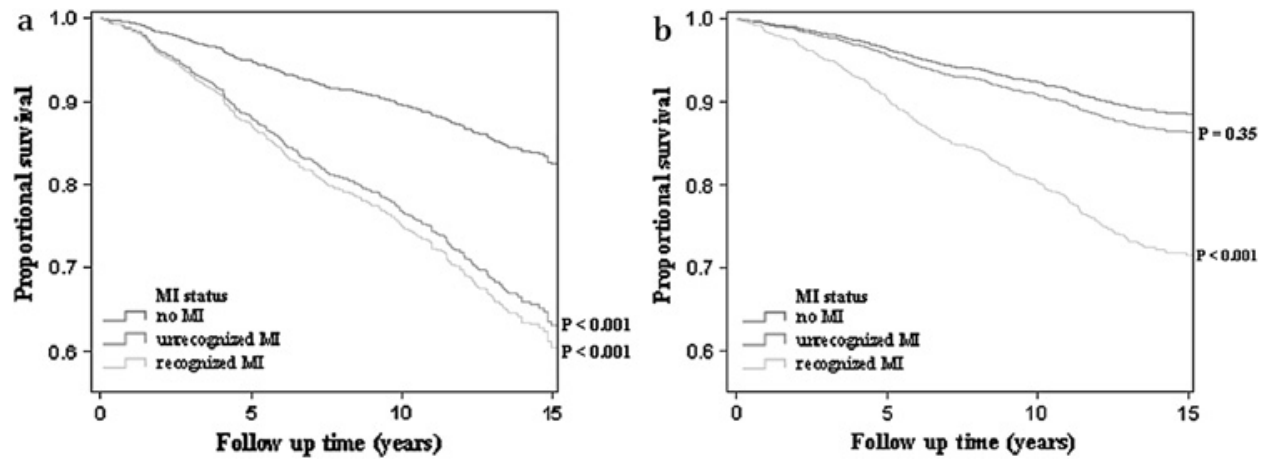

would cause an overestimation of unrecognised MI in women, which is in line with the observed larger proportions of unrecognised MI out of all MI in women (65\%) compared with men $(37 \%){ }^{1-3}{ }^{5-7}$ This line of thought is further supported, to some degree, by the favourable overall cardiovascular risk profile of the women in the unrecognised MI group in comparison with the recognised MI group. The women in the unrecognised MI group were significantly more adipose than women without MI or with recognised $\mathrm{MI}$, with consequently higher possibility of difficulties with ECG interpretation, which also supports this hypothesis (table 1).

\section{Strengths and limitations}

Strengths of our study include the availability of 823 cases of incident heart failure in a population-based setting, with standardised measurements and a long and virtually complete follow-up. Furthermore, at baseline we did not inform the participants or their treating doctors about the finding of an unrecognised MI on their ECG. This reflects the thought at that time that unrecognised MI was less severe than recognised MI, reinforced by lack of evidence that treatment after unrecognised MI could prevent subsequent cardiovascular events. Our study therefore adequately reflects the situation in the general population. During the past few years in the Rotterdam Study, we have started to report findings of unrecognised MI to the participants and treating doctors.

However, our study also has some limitations. First, we chose to examine prevalent $\mathrm{MI}$ to be able to compare the effect of unrecognised MI with recognised MI on the risk of heart failure, since we did not know the actual date of the event of an unrecognised MI. To be able to make a fair comparison between the risks of heart failure after unrecognised MI and after recognised MI, we chose the baseline of our study as the start of follow-up in all participants. Therefore our results only reflect the long-term risk of heart failure. Second, like in all epidemiological studies on unrecognised MI based on ECG, some misclassification may have occurred at baseline. Unrecognised non- $\mathrm{Q}$-wave $\mathrm{MI}$ or those with a $\mathrm{Q}$ wave that has disappeared over time, will not have been detected as unrecognised MI, but classified as no MI.

\section{Clinical implications}

Findings of an unrecognised MI on routine ECG are relatively common in the elderly and our results indicate a comparable long-term risk of heart failure after unrecognised MI and recognised $\mathrm{MI}$ in men. ${ }^{1}$ Our results further suggest that the risk of heart failure after surviving a (un)recognised MI has not substantially decreased over the past two decades, despite great progress in cardiovascular treatment strategies. This merits some concern in a time period in which the rapid ageing of the population' causes overall incidences and costs of heart failure to rise extensively, partially owing to the improved survival after MI. $^{13} 14$

Our results underline the importance of enhanced alertness for atypical symptoms of MI. Doctors should be aware that a large number of patients with unrecognised MI do experience 'sounds of silence': atypical symptoms such as dyspnoea, fatigue or palpitations. ${ }^{29}$ Moreover, patients often misattribute symptoms and their significance. Unrecognised MI may therefore not reflect the heart so much as the perception of the patient. ${ }^{30}$ Better information on typical and atypical symptoms of MI could increase the rate of people seeking medical attention, the first and most essential step in the chain of diagnosis. Even though we did not find a relation between ECG-based unrecognised MI in women and heart failure, we do believe that women as well as men will benefit from a better focus on atypical symptoms of MI.

\section{CONCLUSIONS}

The high incidence of unrecognised MI detected by ECG in the elderly and its equivalent risk of heart failure compared with recognised $\mathrm{MI}$ in men, underlines the importance of enhanced alertness for typical and atypical symptoms of MI by both doctors and patients. The inability to show an association between unrecognised MI in women and incident heart failure adds to the existing suggestion that women are more prone to misclassification of unrecognised MI based on ECG.

Acknowledgements The authors thank all the participants and staff of the Rotterdam Study, as well as the general practitioners and pharmacists of the Ommoord district for help with data collection and validation.

Funding This study is supported by The Netherlands Organisation for Scientific Research (NWO) [VICl 918-76-619]. The Rotterdam Study is supported by Erasmus MC; Erasmus University Rotterdam; Netherlands Organization for Health Research and Development (ZonMw); the Research Institute for Diseases in the Elderly (RIDE); the Ministry of Education, Culture and Science; the Ministry of Health, Welfare and Sports; the European Commission; and the Municipality of Rotterdam.

\section{Competing interests None.}

Patient consent Participants provided written informed consent to participate in the Rotterdam Study and to obtain information from treating doctors.

Ethics approval The study was approved by the medical ethics committee of the Erasmus MC, Rotterdam, The Netherlands.

Provenance and peer review Not commissioned; externally peer reviewed.

\section{REFERENCES}

1. de Torbal A, Boersma E, Kors JA, et al. Incidence of recognized and unrecognized myocardial infarction in men and women aged 55 and older: the Rotterdam Study. Eur Heart J 2006;27:729-36.

2. Boland LL, Folsom AR, Sorlie PD, et al. Occurrence of unrecognized myocardial infarction in subjects aged 45 to 65 years (the ARIC study). Am J Cardiol 2002;90:927-31 
3. Yano K, MacLean CJ. The incidence and prognosis of unrecognized myocardial infarction in the Honolulu, Hawaii, Heart Program. Arch Intern Med 1989;149:1528-32.

4. Sheifer SE, Gersh BJ, Yanez ND 3rd, et al. Prevalence, predisposing factors, and prognosis of clinically unrecognized myocardial infarction in the elderly. J Am Coll Cardiol 2000:35:119-26.

5. Sigurdsson E, Thorgeirsson G, Sigvaldason $\mathrm{H}$, et al. Unrecognized myocardial infarction: epidemiology, clinical characteristics, and the prognostic role of angina pectoris. The Reykjavik Study. Ann Intern Med 1995:122:96-102.

6. Kannel WB, Cupples LA, Gagnon DR. Incidence, precursors and prognosis of unrecognized myocardial infarction. Adv Cardiol 1990;37:202-14.

7. Jonsdottir LS, Sigfusson N, Sigvaldason H, et al. Incidence and prevalence of recognised and unrecognised myocardial infarction in women. The Reykjavik Study. Eur Heart J 1998;19:1011-18.

8. Sigurdsson $\mathbf{E}$, Sigfusson N, Agnarsson U, et al. Long-term prognosis of different forms of coronary heart disease: the Reykjavik Study. Int J Epidemiol 1995;24:58-68.

9. Ikram MA, Hollander M, Bos MJ, et al. Unrecognized myocardial infarction and the risk of stroke: the Rotterdam Study. Neurology 2006;67:1635-9.

10. Ikram MA, van Oijen M, de Jong FJ, et al. Unrecognized myocardial infarction in relation to risk of dementia and cerebral small vessel disease. Stroke 2008;39:1421-6.

11. Lloyd-Jones DM, Larson MG, Leip EP, et al. Lifetime risk for developing congestive heart failure: the Framingham Heart Study. Circulation 2002;106:3068-72.

12. Bleumink GS, Knetsch AM, Sturkenboom MC, et al. Quantifying the heart failure epidemic: prevalence, incidence rate, lifetime risk and prognosis of heart failure The Rotterdam Study. Eur Heart J 2004;25:1614-19.

13. Velagaleti RS, Pencina MJ, Murabito JM, et al. Long-term trends in the incidence of heart failure after myocardial infarction. Circulation 2008:118:2057-62.

14. Lloyd-Jones D, Adams R, Carnethon M, et al. Heart disease and stroke statistics-2009 update: a report from the American Heart Association Statistics Committee and Stroke Statistics Subcommittee. Circulation 2009:119:e21-181.

15. Juenger $\mathbf{J}$, Schellberg $D$, Kraemer $\mathbf{S}$, et al. Health related quality of life in patients with congestive heart failure: comparison with other chronic diseases and relation to functional variables. Heart 2002:87:235-41.
16. Levy D, Kenchaiah $S$, Larson MG, et al. Long-term trends in the incidence of and survival with heart failure. N Engl J Med 2002;347:1397-402.

17. Gottdiener JS, Arnold AM, Aurigemma GP, et al. Predictors of congestive heart failure in the elderly: the Cardiovascular Health Study. J Am Coll Cardiol 2000;35:1628-37.

18. Levy D, Larson MG, Vasan RS, et al. The progression from hypertension to congestive heart failure. JAMA 1996:275:1557-62.

19. He J, Ogden LG, Bazzano LA, et al. Risk factors for congestive heart failure in US men and women: NHANES I epidemiologic follow-up study. Arch Intern Med 2001;161:996-1002

20. Hofman A, Breteler MM, van Duijn CM, et al. The Rotterdam Study: 2010 objectives and design update. Eur J Epidemiol 2009;24:553-72.

21. van Bemmel JH, Kors JA, van Herpen G. Methodology of the modular ECG analysis system MEANS. Methods Inf Med 1990;29:346-53.

22. Prineas RJ, Crow RS, Blackburn H. The Minnesota code manual of electrocardiographic findings. Boston: John Wright PSG, 1982.

23. de Bruyne MC, Mosterd A, Hoes AW, et al. Prevalence, determinants, and misclassification of myocardial infarction in the elderly. Epidemiology 1997:8:495-500

24. Remme WJ, Swedberg K. Task force for the diagnosis and treatment of chronic heart failure ESoC. Guidelines for the diagnosis and treatment of chronic heart failure. Eur Heart J 2001;22:1527-60.

25. Cox DR, Oakes D. Analysysis of survival data. London, UK: Chapman \& Hall, 1984

26. Velagaleti RS, Pencina MJ, Murabito JM, et al. Long-term trends in the incidence of heart failure after myocardial infarction. Circulation 2008;118:2057-62.

27. Dempster AP, Laird NM, Rubin DB. Maximum likelihood from incomplete data via the EM Algorithm. J R Stat Soc B 1977;39:1-38.

28. Murabito JM, Evans JC, Larson MG, et al. Prognosis after the onset of coronary heart disease. An investigation of differences in outcome between the sexes according to initial coronary disease presentation. Circulation 1993;88:2548-55.

29. Stern S. Symptoms other than chest pain may be important in the diagnosis of "silent ischemia," or "the sounds of silence". Circulation 2005;111:e435-7.

30. Barsky AJ, Hochstrasser B, Coles NA, et al. Silent myocardial ischemia. Is the person or the event silent? JAMA 1990:264:1132-5. 